\title{
$4 \pi$-periodic supercurrent tuned by an axial magnetic flux in topological insulator nanowires
}

\author{
Ralf Fischer $\odot,{ }^{1,}{ }^{*}$ Jordi Picó-Cortés $\odot,{ }^{2,3}$ Wolfgang Himmler, ${ }^{1}$ Gloria Platero $\odot,{ }^{2}$ Milena Grifoni $\odot,{ }^{3}$ Dmitriy A. Kozlov, ${ }^{4}$ \\ N. N. Mikhailov, ${ }^{4}$ Sergey A. Dvoretsky $\odot,{ }^{4}$ Christoph Strunk $\odot,{ }^{1}$ and Dieter Weiss ${ }^{1, \dagger}$ \\ ${ }^{1}$ Experimental and Applied Physics, University of Regensburg, D-93040 Regensburg, Germany \\ ${ }_{2}^{2}$ Instituto de Ciencia de Materiales de Madrid (CSIC), 28049 Cantoblanco, Madrid, Spain \\ ${ }^{3}$ Institute of Theoretical Physics, University of Regensburg, D-93040 Regensburg, Germany \\ ${ }^{4}$ A. V. Rzhanov Institute of Semiconductor Physics, Novosibirsk 630090, Russia
}

(Received 22 August 2021; revised 26 November 2021; accepted 30 November 2021; published 3 February 2022)

\begin{abstract}
Topological insulator (TI) nanowires in proximity to conventional superconductors have been proposed as a tunable platform to realize topological superconductivity and Majorana zero modes. The tuning is done using an axial magnetic flux $\phi$ which allows transforming the system from trivial at $\phi=0$ to topologically nontrivial when half a magnetic flux quantum $\phi_{0} / 2$ threads the cross-section of the wire. Here, we explore the expected topological transition in TI-wire-based Josephson junctions as a function of magnetic flux by probing the $4 \pi$ periodic fraction of the supercurrent, which is considered an indicator of topological superconductivity. Our data suggest that this $4 \pi$-periodic supercurrent is at lower magnetic field largely of trivial origin but that, at magnetic fields above $\sim \phi_{0} / 4$, topological $4 \pi$-periodic supercurrents take over.
\end{abstract}

DOI: 10.1103/PhysRevResearch.4.013087

\section{INTRODUCTION}

Majorana zero modes (MZMs), quasiparticle excitations having non-Abelian statistics, were predicted to form at topological superconductor boundaries [1-5] and might be a key component for fault-tolerant quantum computing [6,7]. Different concepts to search for MZMs have been suggested [8]. Semiconductor nanowires with strong spin-orbit interaction in which conventional $s$-wave superconductors induce topological superconductivity have been the prevailing platform to search for MZMs [9-13] (and references therein). These challenging experiments have already provided good signatures for the existence of MZMs at the proximitized region of a semiconductor wire. The drawback is that the Fermi-level $\left(\mu_{\mathrm{F}}\right)$ must be tuned into a material-dependent narrow gap opened by the Zeeman effect and that, despite great efforts, the MZM could not be proven beyond doubt [14]. Recently, $\mathrm{HgTe}$ and InAs quantum wells subjected to an in-plane magnetic field appeared as a platform to search for MZM $[15,16]$. Also, in these experiments, the Zeeman effect is used to tune the system into the topological regime, heralded by a zero-bias peak in experiment.

Here, we explore a conceptually different scheme to realize topological superconductivity by combining $s$-wave superconductors and topological insulator (TI) wires $[17,18]$. The

\footnotetext{
*ralf.fischer@ur.de

†dieter.weiss@ur.de
}

Published by the American Physical Society under the terms of the Creative Commons Attribution 4.0 International license. Further distribution of this work must maintain attribution to the author(s) and the published article's title, journal citation, and DOI. scheme relies on the band structure of a TI wire. Without a magnetic field, the subband structure of the wire features a gap at $k_{x}=0$, with $k_{x}$ the wave vector along the wire. In this state, sketched in Fig. 1(b) (left panel), the band structure is trivial because an even number of modes, say along positive $k$, takes part in transport. Note that every band is degenerate in respect of angular momentum (but not with respect to spin). This changes by applying a magnetic flux of one-half flux quantum $\phi_{0} / 2=h / 2 e(h=$ Planck constant, $e=$ elementary charge) through the cross-section $A$ of the wire. Now the Berry phase, acquired by the spin-momentum locked electron spins around the circumference of the wire, is canceled by the magnetic flux along the wire axis. Therefore, the gap closes, nondegenerate perfectly transmitted surface states appear, and the system becomes topologically nontrivial. The reason is that - for any position of $\mu_{\mathrm{F}}$ in the bulk gap of the TI-the number of propagating modes in one-half of the Brillouin zone is odd [17]. In the case of several occupied subbands, as in our experiments, each (angular momentum resolved) band contributes a single MZM. In this case, an even number of MZMs will pair up and form ordinary fermions, but an odd number of MZMs remains [17].

The subband structure sketched in Fig. 1(b) has been probed by transport experiments in various material systems [19-25]. In contrast to semiconductor wires, where Zeeman splitting is the dominant effect, the energy range for achieving topological superconductivity is much larger in TI wires and not limited to the small Zeeman gap since an odd number of modes exists at $\mu_{\mathrm{F}}$ for any position of $\mu_{\mathrm{F}}$. MZMs in these systems are predicted to be exceptionally stable and present over a wide range of magnetic flux, $\mu_{\mathrm{F}}$, and disorder [18]. This has been confirmed in recent theoretical work that investigated the potential impact of various mechanisms that control the strength of the superconducting proximity effect in quasi-one- 
(a)
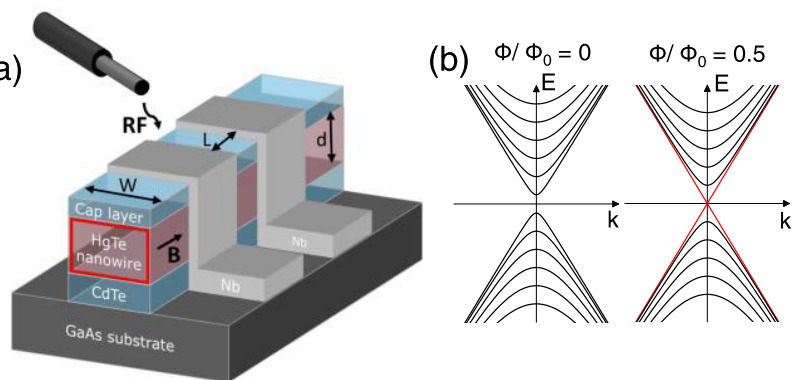

(c)
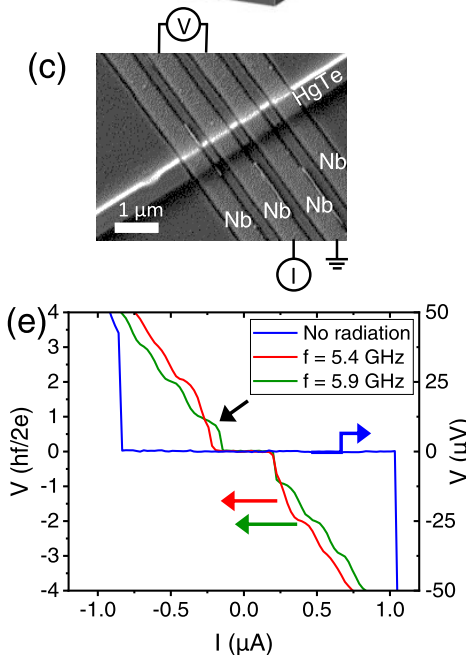

(d) 1
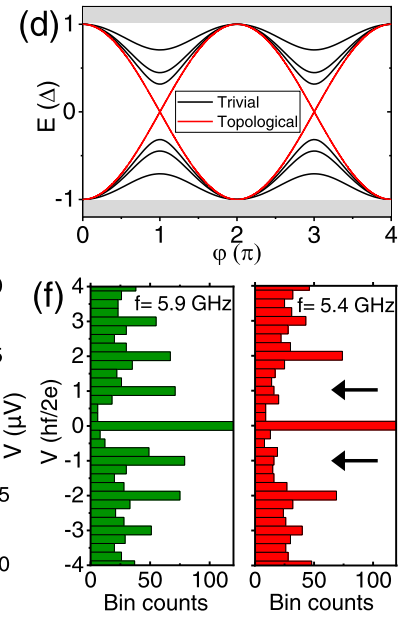

FIG. 1. Sample layout, band structure, and measurement principle. (a) Cartoon of the sample layout showing the HgTe wire and the two $\mathrm{Nb}$ contacts which form the Josephson junction. The "spinless" topological surface states are shown in red. The magnetic field is oriented parallel to the axis of the wires. An antenna near the sample radiates microwaves. (b) Subband structure of the surface states of three-dimensional topological insulator nanowires. For zero magnetic flux $\phi=B w d=0$, the spectrum is gapped with each band degenerate with respect to angular momentum. When $\phi=\phi_{0} / 2$, a gapless, nondegenerate mode appears. (c) Electron micrograph showing the superconducting $\mathrm{Nb}$ stripes placed across the HgTe wire. Only the inner two stripes were used. (d) Spectrum of Andreev bound states with and without Majorana zero mode (MZM). In the trivial case, a gap remains at $\varphi=\pi$, and the energy phase relation is $2 \pi$ periodic. In the topological nontrivial case, MZMs form at $\varphi=\pi$ causing $4 \pi$ periodicity and a $4 \pi$-periodic Josephson current. (e) $I-V$ trace of nanowire $r 1$ (blue trace) at a temperature of $50 \mathrm{mK}$. The junction has a critical current of $I_{\mathrm{C}} \sim 1 \mu \mathrm{A}$. By adding microwave radiation, Shapiro steps appear in the $I-V$ traces (red and green traces). At $5.9 \mathrm{GHz}$, the $n= \pm 1$ steps are visible, while at a lower frequency of $5.4 \mathrm{GHz}$, the first steps are absent (black arrow). (f) Histogram of the data shown in (e).

dimensional TI systems by calculating the dependence of the topological phase diagram and the induced quasiparticle gap on relevant control parameters [26].

To probe signatures of MZMs in our system, we examine the so-called fractional Josephson effect (FJE) where the ac Josephson current should show $4 \pi$ instead of $2 \pi$ periodicity as a function of the phase difference $\varphi$ of the superconductors $[1,2,27]$. MZMs, which live at the boundaries of systems with induced topological superconductivity, have some similarity with Andreev bound states (ABSs) in the induced superconducting gap underneath the superconductor contact. In contrast to ABSs, having an even number of crossings in the interval $[0,2 \pi]$ of the energy-phase relation $[E-\varphi$, see Fig. 1(d)], the presence of MZMs causes a spectrum with an odd number of crossings [2]. As the Josephson current is proportional to $d E(\varphi) / d \varphi$, it becomes $4 \pi$ periodic. In other words, a $2 \pi$-periodic supercurrent flows across the junction in case of conventional Andreev reflection where, upon reflection, a Cooper pair of charge $2 e$ enters (leaves) the superconductor [28]. In contrast, the current becomes $4 \pi$ periodic if only a single elementary charge is transferred. A convenient way to look for the FJE is by measuring the $I-V$ characteristic of the junction under microwave irradiation $[10,29,30]$. With a microwave field, quantized voltage (Shapiro) steps appear at voltages $V_{n}=n h f / 2 e$, with $f$ the microwave frequency and $n$ an integer [31]. In the case of $4 \pi$-periodic Josephson currents, the odd steps, i.e., $n=1,3, \ldots$ are partially missing.

The FJE has been probed, e.g., in two- [30] and threedimensional TIs [29,32,33], Dirac semimetals [34], and semiconductor nanowires $[10,35]$, showing in addition to $2 \pi$ also contributions from $4 \pi$-periodic Josephson currents. The occurrence of $4 \pi$-periodic supercurrents was interpreted as evidence for topological superconductivity. However, missing Shapiro steps have also been found in topologically trivial Josephson junctions [36]. What we contribute here is probing the topological transition in TI-wire-based Josephson junctions with its tunable subband structure by tracing the $4 \pi$ periodic fraction of the supercurrent as a function of magnetic flux. Below, we use axial and perpendicular magnetic fields to disentangle trivial and topological $4 \pi$-periodic currents to explore the trivial-topological transition in our system.

\section{DEVICES, DEVICE PARAMETERS, AND EXPERIMENTAL SETUP}

We fabricated TI wires from strained, $80 \mathrm{~nm} \mathrm{HgTe}$ films, epitaxially grown on $\mathrm{CdTe}$, using e-beam lithography and wet-chemical etching $[25,37,38]$. Figure 1 (a) sketches the device. The $h / e$ periodic conductance oscillations for different $\mu_{\mathrm{F}}$ revealed the topological nature of the surface states in these wires [25]. Wire widths ranged between 250 and $580 \mathrm{~nm}$. Without a gate, $\mu_{\mathrm{F}}$ is typically located at the top of the valence band, so that in addition to Dirac electrons, also bulk holes are present, but the tuning between an even and odd number of modes at $\mu_{\mathrm{F}}$ with an external magnetic field is still viable. Superconducting $\mathrm{Nb}$ contacts were placed on the surface of $\mathrm{HgTe}$ after removing the capping layers by wet-chemical etching. To enhance contact transparency, we cleaned the $\mathrm{HgTe}$ surface by gentle in situ $\mathrm{Ar}^{+}$sputtering. Figure 1(c) displays the electron micrograph of a completed device. Below, we show data from sample $r 1$, which has one of the highest $\mathrm{Nb}$ contact transparencies $D \approx 0.58$ (see Supplemental Material [39], S2). The data from three other samples studied show consistent results. Due to wet-chemical etching, the wires had a trapezoidal cross-section. In the case of wire $r 1$, the top width was $500 \mathrm{~nm}$, the bottom width $580 \mathrm{~nm}$, and the thickness $d=80 \mathrm{~nm}$, resulting in an effective rectangular cross-section of $A=540 \times 80 \mathrm{~nm}$. The circumference of the wires was always shorter than the phase coherence length, 

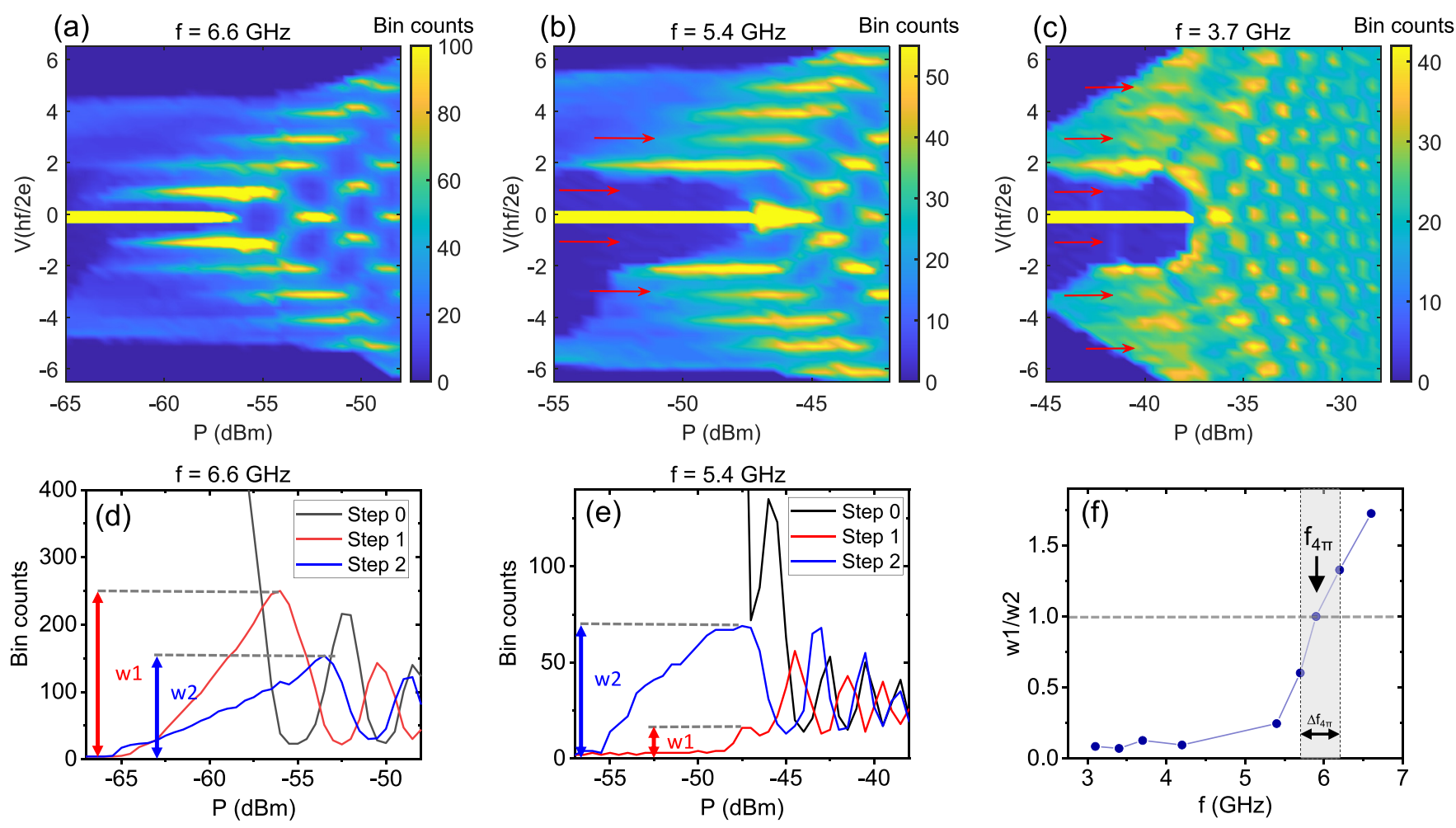

FIG. 2. Frequency dependence of Shapiro steps at $B=0$. (a)-(c) Color map of the bin counts of sample $r 1$ at frequencies $f=6.6,5.4$, and 3.7 GHz. The yellow color marks a step in the $I-V$ traces. For the highest frequency, all Shapiro steps are visible. By lowering the frequency, the first step disappears, and the third one becomes slightly suppressed. Red arrows mark missing or suppressed steps. For the lowest frequency shown, also the third step is strongly suppressed, while the fifth one is further reduced. (d)-(e) Amplitude of the Shapiro steps as a function of the radiation power for the above color map, respectively. At $f=6.6 \mathrm{GHz}$, the maximum amplitude $w 1$ of the first Shapiro step is larger than the second one $w 2$. For $f=5.4 \mathrm{GHz}$, the amplitude of the first step is almost fully suppressed, and the second step dominates. (f) The ratio $w 1 / w 2$ as a function of the frequency. The ratio decreases by lowering the frequency. At the point $w 1 / w 2=1$, the second step starts to dominate. We indicate this point as the transition frequency $f_{4 \pi}$. The grayish background indicates the uncertainty $\Delta f_{4 \pi}$ in the determination of $f_{4 \pi}$.

which was of the order of several microns [25]. Thus, transport within the TI junction is phase coherent. The separation $L$ between adjacent superconducting contacts was between 150 and $170 \mathrm{~nm}$ (wire $r 1$ ). For the topological surface states, this implies a Thouless energy of $\varepsilon_{\mathrm{th}}^{(s)}=\hbar v_{\mathrm{F}}^{(s)} / L \approx 1.6 \mathrm{meV}\left(v_{\mathrm{F}}^{(s)}\right.$ is the Fermi velocity of the surface states) larger than the induced gap $\Delta^{*} \sim 0.59 \mathrm{meV}$ (see Supplemental Material [39], S2). Hence, our devices are in the short junction limit $[40,41]$. The samples were cooled down in a dilution refrigerator with a base temperature of $45 \mathrm{mK}$. The measurements were carried out at this lowest possible temperature to resolve the structures in the $I-V$ characteristics as best as possible. An open-ended coaxial cable, placed a few millimeters near the sample, provided a microwave field with frequencies between 1.5 and $12 \mathrm{GHz}$. The $I-V$ trace with and without applying microwaves is shown in Fig. 1(e). Without microwaves, a supercurrent of $\sim 1 \mu \mathrm{A}$ flows across the junction. With microwaves on, Shapiro steps appear in the $I-V$ trace. For $f=5.9 \mathrm{GHz}$, even and odd voltage steps appear, while at $5.4 \mathrm{GHz}$, the $n=1$ step is missing, and the $n=3$ one is barely visible. To quantify the quality of the steps, we used bar charts, counting the number of data points within a voltage interval around the plateaus. The corresponding plot in Fig. 1(f) (right panel) clearly shows the missing $n=1$ plateau. This indicates a
$4 \pi$-periodic Josephson effect, while the presence of the higher order odd plateaus indicates that conventional $2 \pi$-periodic modes are present [42-44].

\section{SHAPIRO MAPS AT $B=0$}

To be quantitative, we studied in detail the frequency and power dependence, which both affect the Shapiro steps. While the prediction of $4 \pi$-periodic Josephson currents is based on microscopic Hamiltonians, the signature of $I_{2 \pi} \sin (\varphi)$ and $I_{4 \pi} \sin (\varphi / 2)$ supercurrents on the Shapiro step spectrum depend on the normal resistance and capacitance of the junction. The corresponding resistively (RSJ) or resistively and capacitively shunted junction (RCSJ) models permit us to extract the ratio $I_{4 \pi} / I_{2 \pi}$ from frequency- and power-dependent measurements of the Shapiro spectrum $[42,44]$.

Corresponding datasets in Figs. 2(a)-2(c) show colorcoded Shapiro steps measured at three frequencies as a function of power at $B=0$. Increasing microwave power increases the ac current flowing across the junction. From the $I-V$ curves, we extract the histograms [bin counts as in Fig. 1(f)] at fixed power and frequency by dividing the $V$ axis into small intervals of $0.25 h f / 2 e$ and counting the data points within them. The color maps show these histograms 
as a function of microwave power. Yellow regions mark an accumulation of data points and, therefore, a flat line in the $I-V$ traces, i.e., Shapiro steps. Evaluating the maps, we limit ourselves to the low power regime. For higher powers, oscillations appear, which are well described by Bessel functions [45] but not relevant here. For $f=6.6 \mathrm{GHz}$, all Shapiro steps are visible; steps having lower index $n$ appear at lower power. By reducing the frequency to $f=5.4 \mathrm{GHz}$, the first steps $n= \pm 1$ become completely suppressed, and the third ones are slightly reduced. The sequence of all other steps is unchanged. At $f=3.7 \mathrm{GHz}$, the third steps are also fully quenched, while the fifth ones are strongly reduced. So far, only a missing first step was observed [10,29,34], except in Josephson junctions made of two-dimensional HgTe [30]. The absent higher odd index steps $n>1$ show the high quality of our samples and prove that hysteresis, occurring on a smaller bias current scale, is not the origin for the missing steps.

We resort first to a heuristic estimation of $I_{4 \pi}$ based on Ref. [29]. For that, we plot in Figs. 2(d) and 2(e) the power dependency of the histograms of the above Figs. 2(a) and 2(b) at fixed voltages $V_{n}=n h f / 2 e$, for $n=0,1,2$. From these line cuts, we extract the maximum step size (bin count) of the first step $w_{1}$ and the second one $w_{2}$ as shown in the graphs. Figure 2(f) shows the ratio $w_{1} / w_{2}$ as a function of microwave frequency. The ratio $w_{1} / w_{2}$ is nearly constant for frequencies $<5 \mathrm{GHz}$ but rises sharply for higher $f$. The frequency $f_{4 \pi} \approx 5.9 \mathrm{GHz}$ at which $w_{1} / w_{2}=1$ holds is a measure of the amplitude of the $4 \pi$-periodic supercurrent, given by $I_{4 \pi}=\frac{f_{4 \pi} h}{2 e R_{\mathrm{N}}} \approx 57 \mathrm{nA}$, where $R_{\mathrm{N}}$ is the normal-state resistance of $214 \Omega$ [44]. This corresponds to $I_{4 \pi} / I_{C} \approx 6 \%$. Observing a $4 \pi$-periodic supercurrent at $\phi=0$, albeit small, is surprising, as the wire subband structure is topologically trivial. It is conceivable that ballistic trivial modes without gap [46] and/or Landau-Zener transitions between trivial ABSs with a small gap [see Fig. 1(d)] mimic $4 \pi$ periodicity [47]. Indeed, recent experimental work suggests that a few modes with high transparency can undergo Landau-Zener transitions at $\phi=\pi$ and cause $4 \pi$ periodicity [36]. We therefore assume that the missing Shapiro steps at $B=0$ are of trivial origin, i.e., due to Landau-Zener transitions. The observation of a $4 \pi$-periodic Josephson current is thus a necessary but not sufficient proof of topological superconductivity and the presence of MZMs [48]. Below, we show data that trace $I_{4 \pi}$ as a function of magnetic flux from $\phi=0$ to $\phi \sim 0.6 \phi_{0}$, i.e., in a regime where we expect the system to change from trivial to topological. That we observe an increase of $I_{4 \pi} / I_{\mathrm{C}}$ with $\phi$ with a maximum close to $\phi \sim 0.5 \phi_{0}$ is one of the key results of this paper.
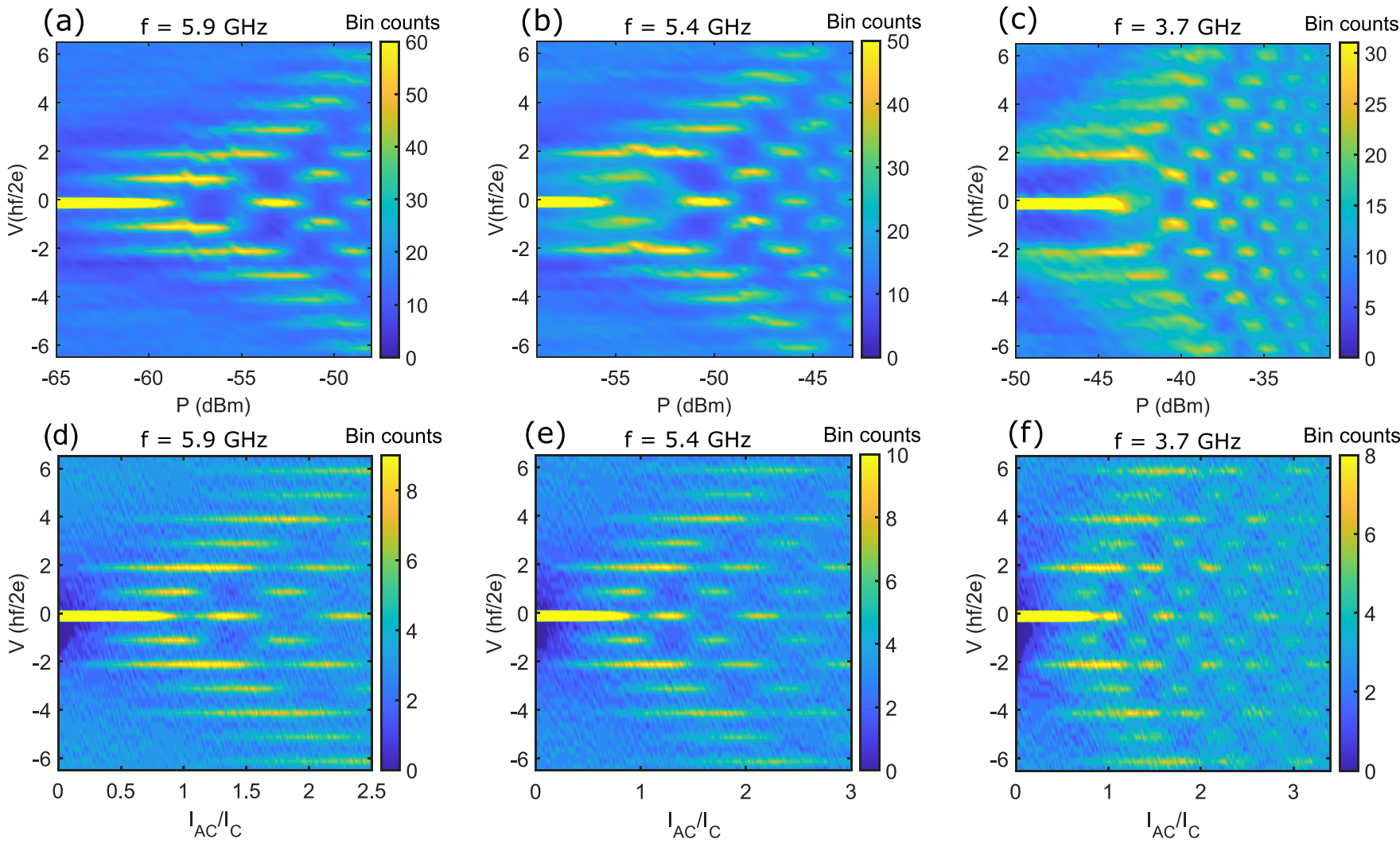

FIG. 3. Frequency dependence of Shapiro steps at $B=30 \mathrm{mT}\left(\phi / \phi_{0} \approx 0.27\right)$. (a)-(c) Color map of the bin counts of sample $r 1$ at frequencies $f=5.9,5.4$, and $3.7 \mathrm{GHz}$. By lowering the frequency, the first step again disappears. However, the transition frequency now slightly changes to $f_{4 \pi}=5.6 \mathrm{GHz}$. With $R_{\mathrm{N}}=216 \Omega$, we estimate $I_{4 \pi} / I_{\mathrm{C}} \approx 21 \%$ using the resistively shunted junction (RSJ) model. (d)-(f) Numerical simulations using the extended resistively and capacitively shunted junction (RCSJ) model. The model additionally includes effective capacitance, Joule heating, and a correction due to the excess current, which leads to $R^{\wedge}=133 \Omega$ (see Supplemental Material [39]). The model almost perfectly fits the experiment by using $I_{4 \pi} / I_{\mathrm{C}} \approx 15.4 \%$ for all frequencies. Minor deviations in the $x$-axis scaling of experiment and calculation occur since the exact relation between $P$ and $I_{\mathrm{AC}}$ is unknown. 

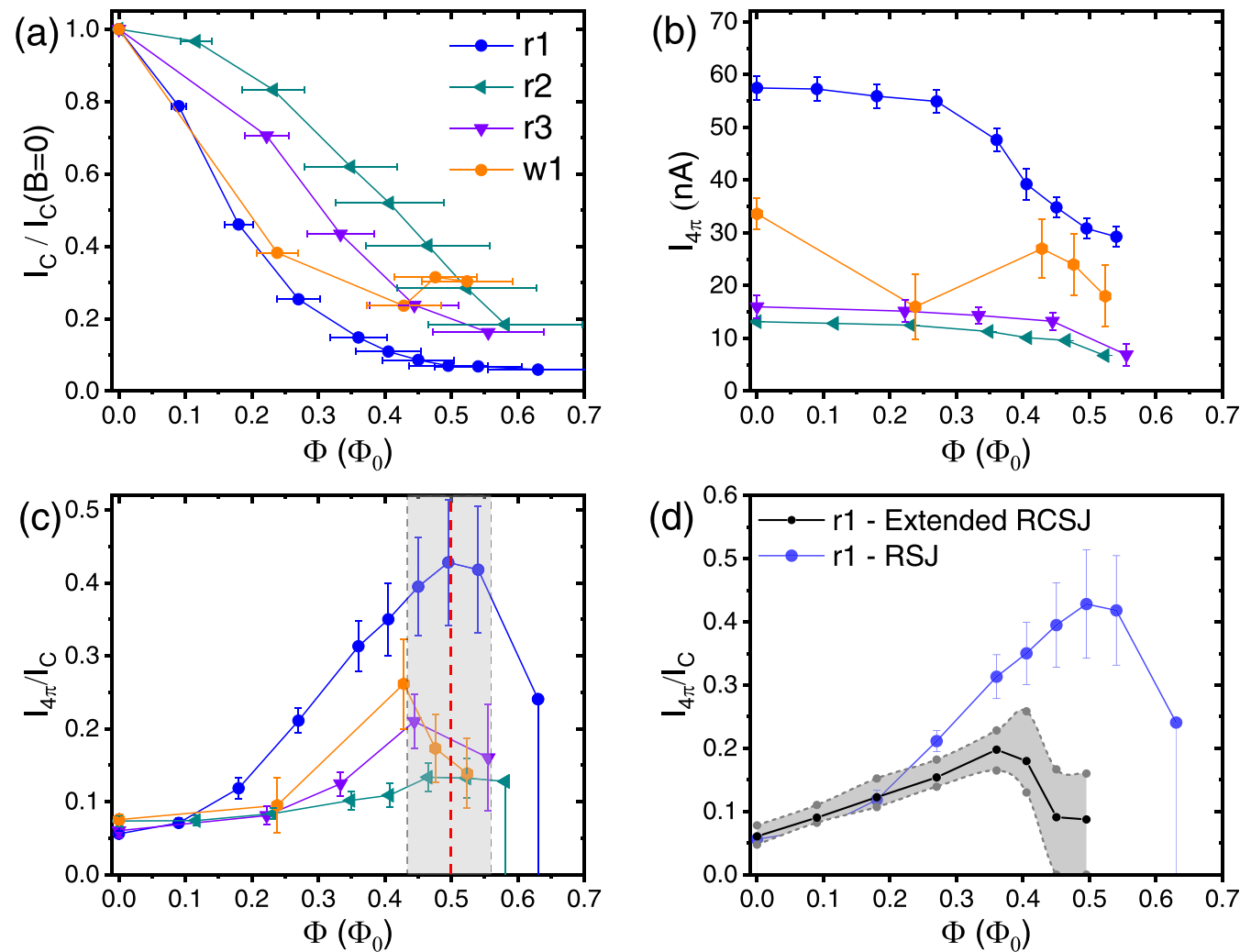

FIG. 4. Magnetic field dependence of the critical currents. (a) The total critical current $I_{\mathrm{C}}$ normalized to its value at $B=0$ for four wires (see Table S1, Supplemental Material [39]) plotted vs the magnetic field applied in parallel to the wire. The amplitude of $I_{\mathrm{C}}$ is strongly reduced by the magnetic field. (b) The $4 \pi$-periodic current $I_{4 \pi}$, in contrast, decreases weaklier as a function of magnetic flux. As in Ref. [29], the resistively shunted junction (RSJ) model has been used to extract $I_{4 \pi}$. (c) The ratio $I_{4 \pi} / I_{\mathrm{C}}$ obtained from the RSJ model plotted as a function of the magnetic field. The red line marks $\phi / \phi_{0}=0.5$, while the grayish background indicates its uncertainty, as the cross-sectional area can only be determined within $\sim 12 \%$. All traces display maxima at about $\phi / \phi_{0} \sim 0.5$. For wire $r 1$, this corresponds to $B \sim 55.5 \mathrm{mT}$. (d) Comparison of $I_{4 \pi} / I_{\mathrm{C}}$ extracted from wire $r 1$ using the RSJ model and simulations employing the extended resistively and capacitively shunted junction (RCSJ) model. The ratio $I_{4 \pi} / I_{\mathrm{C}}$ is extracted by comparing the experimental and simulated results (see Supplemental Material [39]). The black dots resemble the values for which the simulations fit the experimental data most accurately, while ratios in the gray area also lead to satisfying results and can be regarded as error bars. The extended RCSJ model gives somewhat smaller values at high magnetic fields than the heuristic estimation but also shows a distinct maximum, which occurs at a lower field. However, the exact position of the maximum in the blue curve is hard to determine due to the large error bars around $\phi / \phi_{0} \sim 0.5$.

\section{SHAPIRO MAPS WITH AXIAL MAGNETIC FLUX}

In Figs. 3(a)-3(c), we show an example of the data measured at $B=30 \mathrm{mT}$, aligned along the wire axis and corresponding to $\phi=B A \approx 0.27 \phi_{0}$. The first Shapiro steps, fully present at $5.9 \mathrm{GHz}$, are substantially weakened at $5.4 \mathrm{GHz}$ and are fully absent below $f=3.7 \mathrm{GHz}$. Thus, compared with $B=0$, the crossover frequency $f_{4 \pi}$ is shifted to slightly lower frequencies. We obtain $I_{4 \pi}=\frac{f_{4 \pi} h}{2 e R_{N}} \approx 55 \mathrm{nA}$, slightly smaller than without $B$. However, as $I_{\mathrm{C}}$ has decreased much more with $B$ to $I_{\mathrm{C}}=260 \mathrm{nA}$, a larger fraction of the supercurrent $I_{4 \pi} / I_{\mathrm{C}} \sim 0.21$ is carried by the $4 \pi$ contribution. Figures 3(d)-3(f) are calculations within the RCSJ model addressed below.

A detailed overview of the $B$ dependence of $I_{\mathrm{C}}$ and $I_{4 \pi}$ of four samples is displayed in Figs. 4(a) and 4(b). Figure 4(a) shows that the critical current $I_{\mathrm{C}}$ drops quickly with increasing $\phi$, while $I_{4 \pi}$ in Fig. 4(b) decreases much less. The corresponding ratio $I_{4 \pi} / I_{\mathrm{C}}$ is plotted in Fig. 4(c), displaying a maximum when about half a flux quantum $\phi_{0} / 2$ threads the trapezoidal cross-section of the wires. The effect is largest for wire $r 1$. For $r 1, I_{4 \pi} / I_{\mathrm{C}}$ increases from $\sim 6 \%$ at $B=0$ to $43 \%$ at $B=55 \mathrm{mT}$. Upon further increase of $B$, we expect the perfectly transmitted state to disappear again and $I_{4 \pi} / I_{\mathrm{C}}$ to drop. At $B \sim 70 \mathrm{mT}\left(\phi / \phi_{0} \sim 0.63\right)$, we observe no missing Shapiro steps anymore down to $f=1.5 \mathrm{GHz}$, which is the lowest frequency at which Shapiro steps get resolved. The blue point of wire $r 1$ in Fig. 4(c) at $\phi / \phi_{0} \sim 0.63$ can be regarded as an upper limit for $I_{4 \pi} / I_{\mathrm{C}}$. If just below $f=1.5 \mathrm{GHz}$, odd Shapiro steps start to disappear, and assigning $f_{4 \pi} \sim 1.5$ $\mathrm{GHz}$, we arrive at $I_{4 \pi} / I_{\mathrm{C}} \sim 24 \%$. Note though that $I_{4 \pi} / I_{\mathrm{C}}$ might be significantly lower, as is indicated by the large error bar. In addition to $r 1$, the data of three other samples with different geometries are shown in Figs. 4(a)-4(c). The properties of these samples can be found in the Supplemental Material [39], S2. All samples show a similar dependence of $I_{4 \pi} / I_{\mathrm{C}}$ on the flux $\phi$, although the peak height near $\phi / \phi_{0} \sim 0.5$ varies strongly. This variation is most likely linked to the $B$ dependence of the $I_{2 \pi}$-periodic current, which dominates the critical current $I_{\mathrm{C}}$. That $I_{4 \pi} / I_{\mathrm{C}}(B)$ has a maximum sometimes 
slightly below $\phi=\phi_{0} / 2$ is expected as this, together with the $B$ range in which the perfectly transmitted mode occurs, depends on many experimental parameters, like the exact position of $\mu_{\mathrm{F}}$, disorder, or the exact shape of the nanowire [49]. It also depends on the distribution of transparencies between the superconductor and individual transport channels [26].

\section{MODELING THE EXPERIMENTAL DATA WITH THE RCSJ MODEL}

So far, we have estimated $I_{4 \pi}$ using a heuristic approach based on the RSJ model, which neglects several effects that may influence the observed Shapiro signatures. In addition, we have performed numerical simulations based on an extended RCSJ model aiming to reproduce the experimental results as accurately as possible. Details of the model can be found in the Supplemental Material [39], S3. The results for $B=30 \mathrm{mT}$ are presented in Figs. 3(d)-3(f) (the results for other $B$ values are shown in the Supplemental Material [39], S6). As shown there, the theoretical model accurately predicts the Shapiro response of the junction as a function of radiofrequency power and frequency. Aiming to match the experimental data, the results include an ef- fective capacitance, which we estimate based on the model of Ref. [50] (see Supplemental Material [39], S2 and S5). The model also takes excess currents in the samples into account. Excess currents affect the estimation of $I_{4 \pi}$ by altering the effective resistance at small voltages as compared with its normal state value. From the measured $I-V$ curves (see Fig. S1(a) in the Supplemental Material [39]), we see that the resistance at $V \simeq 0$ ranges from $96 \Omega$ at $B=0$ to 157 $\Omega$ at $B=50 \mathrm{mT}$, which would yield a larger value for $I_{4 \pi}$ than estimated. On the other hand, the RSJ model is known to overestimate $I_{4 \pi}$ compared with the capacitively shunted model [43]. Moreover, the model includes thermal effects due to Joule heating in the sample by involving the dissipation of the applied electric power via the electron-phonon coupling (see Supplemental Material [39], S4). This allows us to rate the temperature dependence of the different parameters. The simulated values are presented in Fig. 4(d). Comparing the RSJ with the RCSJ traces in Fig. 4(d) shows that the value of $I_{4 \pi} / I_{\mathrm{C}}$ depends strongly on the underlying model. Both analysis methods, however, show qualitative agreement, i.e., an increase of $I_{4 \pi} / I_{\mathrm{C}}$ with increasing flux with a maximum near half flux quantum. Theoretically, the exact position of this maximum (transition) depends on several factors [26] and may occur before $\phi_{0} / \phi=0.5$.

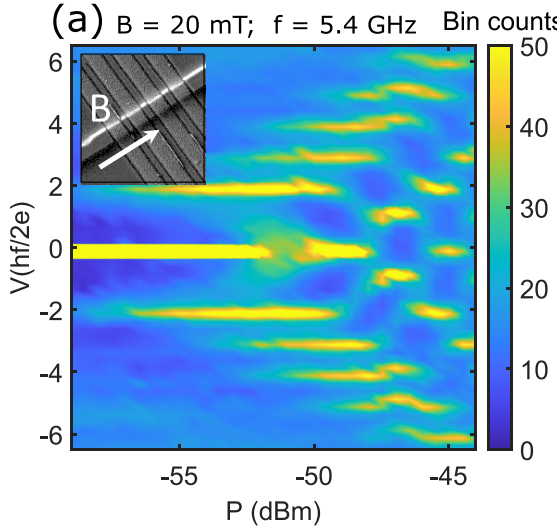

(d) $\mathrm{B}=20 \mathrm{mT} ; \mathrm{f}=5.4 \mathrm{GHz}$ Bin counts

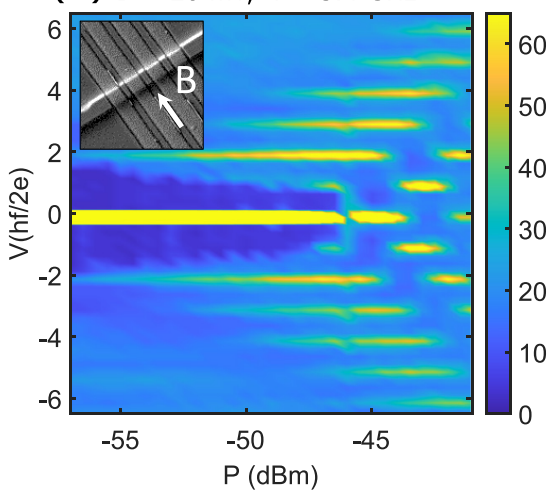

(b) $\mathrm{B}=40 \mathrm{mT} ; \mathrm{f}=3.7 \mathrm{GHz}$ Bin counts

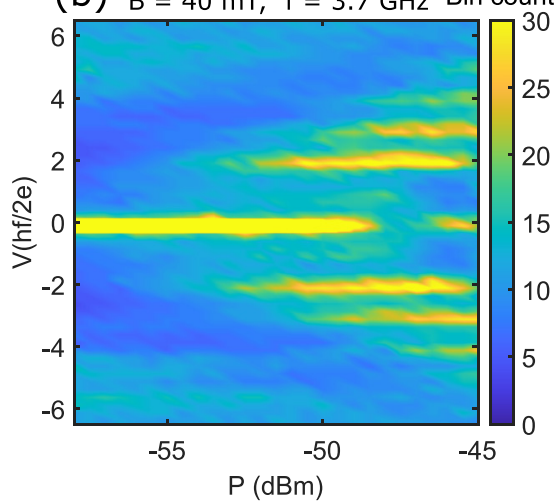

(e) $B=40 \mathrm{mT} ; f=3.7 \mathrm{GHz}$ Bin counts

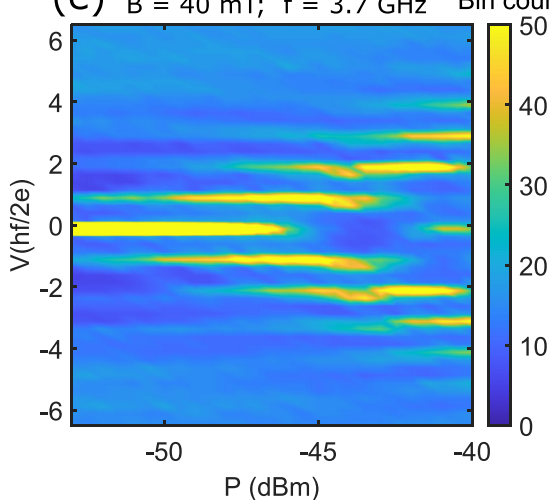

(c) $\mathrm{B}=45 \mathrm{mT} ; \mathrm{f}=3.1 \mathrm{GHz}$ Bin counts

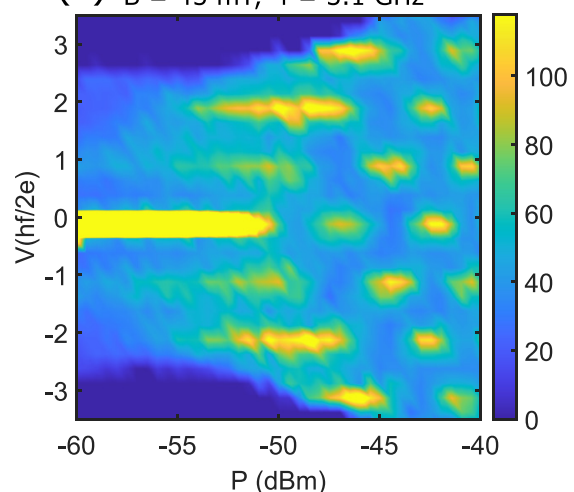

(f) $B=45 \mathrm{mT} ; \mathrm{f}=3.1 \mathrm{GHz}$ Bin counts

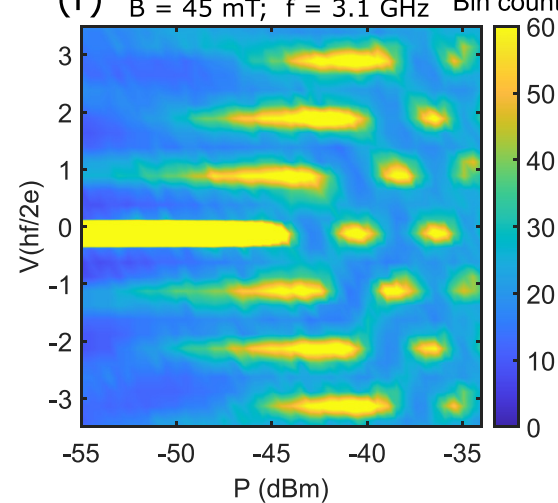

FIG. 5. Shapiro maps at magnetic fields parallel and perpendicular to the wire. (a)-(c) $B$ parallel to the wire (sample $r 1$ ) as shown in the inset of (a). Color maps of the bin counts at $B=20 \mathrm{mT}$ and $f=5.4 \mathrm{GHz}, B=40 \mathrm{mT}$ and $f=3.7 \mathrm{GHz}$, and $B=45 \mathrm{mT}$ and $f=3.1 \mathrm{GHz}$. The first step is strongly quenched or fully absent at these frequencies for the respective magnetic field values. (d)-(f) $B$ perpendicular to the same wire as shown in the inset of (d). Color map of the bin counts at $B_{\perp}=20 \mathrm{mT}$ and $f=5.4 \mathrm{GHz}, B_{\perp}=40 \mathrm{mT}$ and $f=3.7 \mathrm{GHz}$, and $B_{\perp}=45 \mathrm{mT}$ and $f=3.1 \mathrm{GHz}$. While in (d) the first step is quenched like in (a), in (e) and (f), all steps are fully visible. The latter is contrary to the observations in (b) and (c). This indicates an additional $4 \pi$-periodic component of the supercurrent, which is only present for a magnetic field $B>20-30 \mathrm{mT}$, which is oriented along the wire. 


\section{DISTINCTION BETWEEN TRIVIAL AND TOPOLOGICAL $4 \pi$-PERIODIC SUPERCURRENTS}

Despite the excellent agreement between theory and experiment and the maxima of $I_{4 \pi} / I_{\mathrm{C}}$ around $\phi_{0} / \phi=0.5$, we need to distinguish between trivial (e.g., via Landau-Zener transitions) and topological origin of the $4 \pi$-periodic supercurrent. To clarify this, we have measured the Shapiro maps in an axial and perpendicular magnetic field as shown in Figs. 5(a) and 5(d). For perpendicular $B$, the system is trivial, and no $I_{4 \pi}$ supercurrent of topological origin is expected. The decrease of $I_{\mathrm{C}}$ with $B$ is in both configurations quite similar (see Supplemental Material [39], S7), which allows us to directly compare the Shapiro maps at specific magnetic field values. Corresponding data for parallel (perpendicular) $B$ are shown in the upper (lower) row of Fig. 5. At $20 \mathrm{mT}$ at $f=5.4 \mathrm{GHz}$, the first Shapiro step is suppressed in both configurations. Thus, we expect a similar transition frequency $f_{4 \pi}$ and $4 \pi$-periodic current $I_{4 \pi}$. This suggests that, in this low field range, $I_{4 \pi}$ is largely of trivial origin. At higher $B$, the situation changes. For $B=40 \mathrm{mT}$ at $f=3.7 \mathrm{GHz}$ and for $B=45 \mathrm{mT}$ at $f=3.1 \mathrm{GHz}$, the first Shapiro step is suppressed for parallel configuration [Figs. 5(b) and 5(c)] while fully present if $B$ is oriented perpendicular to the wire. Thus, for $B>30 \mathrm{mT}, I_{4 \pi}$ is consistently lower for perpendicular configuration. These observations can be explained by trivial $4 \pi$-periodic contributions, which get suppressed for both configurations with increasing $B$, while the topological perfectly transmitted mode emerges with increasing $B$. This suggests that the $4 \pi$-periodic supercurrent is approximately in the interval $0.25<\phi / \phi_{0}<0.5$ of topological origin. This is also consistent with the decrease of $I_{4 \pi} / I_{\mathrm{C}}(B)$ in Fig. 4(d) for $\phi / \phi_{0}>0.5$, as the topological $4 \pi$-periodic contribution is expected to vanish again.

\section{CONCLUSIONS}

To summarize, our data and simulation suggest that, around $\phi_{0} / \phi \sim 0.5$, the observed $4 \pi$-periodic supercurrent is largely of topological origin. Our experiments are hence a first indication that one can indeed switch between trivial and topological superconductivity with an axial magnetic flux in TI wires, as suggested theoretically $[17,51,52]$.

\section{ACKNOWLEDGMENTS}

This paper was funded by the European Research Council under the European Union's Horizon 2020 research and innovation program (Grant Agreement No. 787515, 253 ProMotion). R.F. obtained funding from the Elite Network of Bavaria (K-NW-2013-258, Doctorate Program "Topological Insulators"). D.A.K. was supported by the Ministry of Science and Higher Education of the Russian Federation, Grant No. 075-15-2020-797 (13.1902.21.0024). N.N.M. and S.A.D. acknowledge support by RFBR Grant 18-29-20053. M.G. acknowledges support from the German Research Foundation (DFG) Project-ID 314695032-CRC 1277 (Subproject B04). G.P. acknowledges the Mercator Fellow position by the DFG via CRC 1277 in Regensburg, as well as Spain's MINECO through Grants No. MAT2017-86717-P, No. PID2020-117787GB-100 and the CSIC Research Platform PTI-001.
[1] A. Y. Kitaev, Unpaired Majorana fermions in quantum wires, Phys. Usp. 44, 131 (2001).

[2] R. M. Lutchyn, J. D. Sau, and S. Das Sarma, Majorana Fermions and a Topological Phase Transition in Semiconductor-Superconductor Heterostructures, Phys. Rev. Lett. 105, 077001 (2010).

[3] Y. Oreg, G. Refael, and F. von Oppen, Helical Liquids and Majorana Bound States in Quantum Wires, Phys. Rev. Lett. 105, 177002 (2010).

[4] J. Alicea, New directions in the pursuit of Majorana fermions in solid state systems, Rep. Prog. Phys. 75, 076501 (2012).

[5] C. W. J. Beenakker, Search for Majorana fermions in superconductors, Annu. Rev. Condens. Matter Phys. 4, 113 (2013).

[6] A. Y. Kitaev, Fault-tolerant quantum computation by anyons, Ann. Phys. 303, 2 (2003).

[7] C. Nayak, S. H. Simon, A. Stern, M. Freedman, and S. Das Sarma, Non-Abelian anyons and topological quantum computation, Rev. Mod. Phys. 80, 1083 (2008).

[8] J. Alicea, Majorana fermions in a tunable semiconductor device, Phys. Rev. B 81, 125318 (2010).

[9] V. Mourik, K. Zuo, S. M. Frolov, S. R. Plissard, E. P. A. M. Bakkers, and L. P. Kouwenhoven, Signatures of Majorana fermions in hybrid superconductor-semiconductor nanowire devices, Science 336, 1003 (2012).
[10] L. P. Rokhinson, X. Liu, and J. K. Furdyna, The fractional a.c. Josephson effect in a semiconductor-superconductor nanowire as a signature of Majorana particles, Nat. Phys. 8, 795 (2012).

[11] M. T. Deng, S. Vaitiekènas, E. B. Hansen, J. Danon, M. Leijnse, K. Flensberg, J. Nygård, P. Krogstrup, and C. M. Marcus, Majorana bound state in a coupled quantum-dot hybrid-nanowire system, Science 354, 1557 (2016).

[12] S. M. Albrecht, A. P. Higginbotham, M. Madsen, F. Kuemmeth, T. S. Jespersen, J. Nygård, P. Krogstrup, and C. M. Marcus, Exponential protection of zero modes in Majorana islands, Nature (London) 531, 206 (2016).

[13] Ö. Gül, H. Zhang, J. D. S. Bommer, M. W. A. de Moor, D. Car, S. R. Plissard, E. P. A. M. Bakkers, A. Geresdi, K. Watanabe, T. Taniguchi, and L. P. Kouwenhoven, Ballistic Majorana nanowire devices, Nat. Nanotechnol. 13, 192 (2018).

[14] H. Zhang, M. W. A. de Moor, J. D. S. Bommer, D. Xu, G. Wang, N. van Loo, C.-X. Liu, S. Gazibegovic, J. A. Logan, D. Car, R. L. M. Op het Veld, P. J. van Veldhoven, S. Koelling, M. A. Verheijen, M. Pendharkar, D. J. Pennachio, B. Shojaei, J. S. Lee, C. J. Palmstrøm, E. P. A. M. Bakkers et al., Large zerobias peaks in InSb-Al hybrid semiconductor-superconductor nanowire devices, arXiv:2101.11456.

[15] H. Ren, F. Pientka, S. Hart, A. T. Pierce, M. Kosowsky, L. Lunczer, R. Schlereth, B. Scharf, E. M. Hankiewicz, L. W. 
Molenkamp, B. I. Halperin, and A. Yacoby, Topological superconductivity in a phase-controlled Josephson junction, Nature (London) 569, 93 (2019).

[16] A. Fornieri, A. M. Whiticar, F. Setiawan, E. Portolés, A. C. C. Drachmann, A. Keselman, S. Gronin, C. Thomas, T. Wang, R. Kallaher, G. C. Gardner, E. Berg, M. J. Manfra, A. Stern, C. M. Marcus, and F. Nichele, Evidence of topological superconductivity in planar Josephson junctions, Nature (London) 569, 89 (2019).

[17] A. Cook and M. Franz, Majorana fermions in a topologicalinsulator nanowire proximity-coupled to an $s$-wave superconductor, Phys. Rev. B 84, 201105(R) (2011).

[18] A. M. Cook, M. M. Vazifeh, and M. Franz, Stability of Majorana fermions in proximity-coupled topological insulator nanowires, Phys. Rev. B 86, 155431 (2012).

[19] M. Tian, W. Ning, Z. Qu, H. Du, J. Wang, and Y. Zhang, Dual evidence of surface Dirac states in thin cylindrical topological insulator $\mathrm{Bi}_{2} \mathrm{Te}_{3}$ nanowires, Sci. Rep. 3, 1212 (2013).

[20] J. Dufouleur, L. Veyrat, A. Teichgräber, S. Neuhaus, C. Nowka, S. Hampel, J. Cayssol, J. Schumann, B. Eichler, O. G. Schmidt, B. Büchner, and R. Giraud, Quasiballistic Transport of Dirac Fermions in a $\mathrm{Bi}_{2} \mathrm{Se}_{3}$ Nanowire, Phys. Rev. Lett. 110, 186806 (2013).

[21] M. Safdar, Q. Wang, M. Mirza, Z. Wang, K. Xu, and J. He, Topological surface transport properties of single-crystalline SnTe nanowire, Nano Lett. 13, 5344 (2013).

[22] S. S. Hong, Y. Zhang, J. J. Cha, X.-L. Qi, and Y. Cui, Onedimensional helical transport in topological insulator nanowire interferometers, Nano Lett. 14, 2815 (2014).

[23] S. Cho, B. Dellabetta, R. Zhong, J. Schneeloch, T. Liu, G. Gu, M. J. Gilbert, and N. Mason, Aharonov-Bohm oscillations in a quasi-ballistic three-dimensional topological insulator nanowire, Nat. Commun. 6, 7634 (2015).

[24] L. A. Jauregui, M. T. Pettes, L. P. Rokhinson, L. Shi, and Y. P. Chen, Magnetic field-induced helical mode and topological transitions in a topological insulator nanoribbon, Nat. Nanotechnol. 11, 345 (2016).

[25] J. Ziegler, R. Kozlovsky, C. Gorini, M. H. Liu, S. Weishäupl, H. Maier, R. Fischer, D. A. Kozlov, Z. D. Kvon, N. Mikhailov, S. A. Dvoretsky, K. Richter, and D. Weiss, Probing spin helical surface states in topological HgTe nanowires, Phys. Rev. B 97, 035157 (2018).

[26] P. Sitthison and T. D. Stanescu, Robustness of topological superconductivity in proximity-coupled topological insulator nanoribbons, Phys. Rev. B 90, 035313 (2014).

[27] H. J. Kwon, K. Sengupta, and V. M. Yakovenko, Fractional ac Josephson effect in $p$ - and $d$-wave superconductors, Eur. Phys. J. B 37, 349 (2004).

[28] B. Pannetier and H. Courtois, Andreev reflection and proximity effect, J. Low Temp. Phys. 118, 599 (2000).

[29] J. Wiedenmann, E. Bocquillon, R. S. Deacon, S. Hartinger, O. Herrmann, T. M. Klapwijk, L. Maier, C. Ames, C. Brüne, C. Gould, A. Oiwa, K. Ishibashi, S. Tarucha, H. Buhmann, and L. W. Molenkamp, $4 \pi$-periodic Josephson supercurrent in HgTe-based topological Josephson junctions, Nat. Commun. 7, 10303 (2016).

[30] E. Bocquillon, R. S. Deacon, J. Wiedenmann, P. Leubner, T. M. Klapwijk, C. Brüne, K. Ishibashi, H. Buhmann, and L. W. Molenkamp, Gapless Andreev bound states in the quantum spin Hall insulator HgTe, Nat. Nanotechnol. 12, 137 (2017).
[31] S. Shapiro, Josephson Currents in Superconducting Tunneling: The Effect of Microwaves and Other Observations, Phys. Rev. Lett. 11, 80 (1963).

[32] R. S. Deacon, J. Wiedenmann, E. Bocquillon, F. Domínguez, T. M. Klapwijk, P. Leubner, C. Brüne, E. M. Hankiewicz, S Tarucha, K. Ishibashi, H. Buhmann, and L. W. Molenkamp, Josephson Radiation from Gapless Andreev Bound States in HgTe-Based Topological Junctions, Phys. Rev. X 7, 021011 (2017).

[33] P. Schüffelgen, D. Rosenbach, C. Li, T. W. Schmitt, M Schleenvoigt, A. R. Jalil, S. Schmitt, J. Kölzer, M. Wang, B. Bennemann, U. Parlak, L. Kibkalo, S. Trellenkamp, T. Grap, D. Meertens, M. Luysberg, G. Mussler, E. Berenschot, N. Tas, A. A. Golubov et al., Selective area growth and stencil lithography for in situ fabricated quantum devices, Nat. Nanotechnol. 14, 825 (2019).

[34] C. Li, J. C. de Boer, B. de Ronde, S. V. Ramankutty, E. van Heumen, Y. Huang, A. de Visser, A. A. Golubov, M. S. Golden, and A. Brinkman, $4 \pi$-periodic Andreev bound states in a Dirac semimetal, Nat. Mater. 17, 875 (2018).

[35] D. Laroche, D. Bouman, D. J. van Woerkom, A. Proutski, C. Murthy, D. I. Pikulin, C. Nayak, R. J. J. van Gulik, J. Nygård, P. Krogstrup, L. P. Kouwenhoven, and A. Geresdi, Observation of the $4 \pi$-periodic Josephson effect in indium arsenide nanowires, Nat. Commun. 10, 245 (2019).

[36] M. C. Dartiailh, J. J. Cuozzo, B. H. Elfeky, W. Mayer, J. Yuan, K. S. Wickramasinghe, E. Rossi, and J. Shabani, Missing Shapiro steps in topologically trivial Josephson junction on InAs quantum well, Nat. Commun. 12, 78 (2021).

[37] D. A. Kozlov, Z. D. Kvon, E. B. Olshanetsky, N. N Mikhailov, S. A. Dvoretsky, and D. Weiss, Transport Properties of a 3D Topological Insulator based on a Strained High-Mobility HgTe Film, Phys. Rev. Lett. 112, 196801 (2014).

[38] D. A. Kozlov, D. Bauer, J. Ziegler, R. Fischer, M. L. Savchenko, Z. D. Kvon, N. N. Mikhailov, S. A. Dvoretsky, and D. Weiss, Probing Quantum Capacitance in a 3D Topological Insulator, Phys. Rev. Lett. 116, 166802 (2016).

[39] See Supplemental Material at http://link.aps.org/supplemental/ 10.1103/PhysRevResearch.4.013087 for subband structure of the TI wires, sample fabrication and properties of the fabricated junctions, the extended RCSJ model, estimation of the intrinsic capacitance, comparison between additional theoretical simulations and experiments, and Shapiro maps in perpendicular magnetic fields.

[40] D. Averin and A. Bardas, ac Josephson Effect in a Single Quantum Channel, Phys. Rev. Lett. 75, 1831 (1995).

[41] J. C. Cuevas, A. Martín-Rodero, and A. Levy Yeyati, Hamiltonian approach to the transport properties of superconducting quantum point contacts, Phys. Rev. B 54, 7366 (1996).

[42] F. Domínguez, O. Kashuba, E. Bocquillon, J. Wiedenmann, R. S. Deacon, T. M. Klapwijk, G. Platero, L. W. Molenkamp, B. Trauzettel, and E. M. Hankiewicz, Josephson junction dynamics in the presence of $2 \pi$ - and $4 \pi$-periodic supercurrents, Phys. Rev. B 95, 195430 (2017).

[43] J. Picó-Cortés, F. Domínguez, and G. Platero, Signatures of a $4 \pi$-periodic supercurrent in the voltage response of capac- 
itively shunted topological Josephson junctions, Phys. Rev. B 96, 125438 (2017).

[44] F. Domínguez, F. Hassler, and G. Platero, Dynamical detection of Majorana fermions in current-biased nanowires, Phys. Rev. B 86, 140503(R) (2012).

[45] P. Russer, Influence of microwave radiation on current-voltage characteristic of superconducting weak links, J. Appl. Phys. 43, 2008 (1972).

[46] C. W. J. Beenakker, Universal Limit of Critical-Current Fluctuations in Mesoscopic Josephson Junctions, Phys. Rev. Lett. 67, 3836 (1991).

[47] P. M. Billangeon, F. Pierre, H. Bouchiat, and R. Deblock, ac Josephson Effect and Resonant Cooper Pair Tunneling Emission of a Single Cooper Pair Transistor, Phys. Rev. Lett. 98, 216802 (2007).
[48] C.-K. Chiu and S. Das Sarma, Fractional Josephson effect with and without Majorana zero modes, Phys. Rev. B 99, 035312 (2019).

[49] F. de Juan, J. H. Bardarson, and R. Ilan, Conditions for fully gapped topological superconductivity in topological insulator nanowires, SciPost Phys. 6, 060 (2019).

[50] D. S. Antonenko and M. A. Skvortsov, Quantum decay of the supercurrent and intrinsic capacitance of Josephson junctions beyond the tunnel limit, Phys. Rev. B 92, 214513 (2015).

[51] F. de Juan, R. Ilan, and J. H. Bardarson, Robust Transport Signatures of Topological Superconductivity in Topological Insulator Nanowires, Phys. Rev. Lett. 113, 107003 (2014).

[52] T. D. Stanescu and S. Tewari, Majorana fermions in semiconductor nanowires: fundamentals, modeling, and experiment, J. Phys.: Condens. Matter 25, 233201 (2013). 